\title{
La mirada interpretativa como base de una discrecionalidad discreta*
}

\author{
The interpretative view as the basis \\ of a discreet thesis about judicial discretion
}

\section{O olhar interpretativo como base de discrição discreta}

\section{Diego Gamarra ${ }^{1}$}

${ }^{1}$ Universidad Católica del Uruguay

Orcid 0000-0003-0902-786X

DOI: https://doi.org/10.22235/rd.v0i19.1731

Comunicación

ReSUMEN: En la senda trazada por Dworkin, se propone una concepción sobre la discrecionalidad jurisdiccional -discreta- a partir de la asunción de una necesaria posición interpretativa. A esos efectos se parte de la tesis de la indeterminación parcial del lenguaje y en particular de las reglas -conforme la formulación de Hart de tal posición-, que en términos de aplicación del derecho, independientemente de la perspectiva,

Recibido: 06/12/18 Evaluado: 14/02/19. Aceptado: 17/02/19.

* Esta comunicación es una reelaboración de algunos puntos sostenidos por el autor en su tesis doctoral, (Los jueces entre la Constitución y la ley. Interpretación jurisdiccional de disposiciones constitucionales y legales concurrente, UCM, 2016), preparada para ser publicada en este número especial de Revista de Derecho.

Diego Gamarra Ante: Abogado (UCU), master en Derecho parlamentario y doctor en Derecho (ambos, Universidad Complutense de Madrid). Profesor de Derecho Constitucional y director del Departamento de Derecho Público, Universidad Católica del Uruguay. Miembro de la Asociación Uruguaya de Derecho Constitucional y de Derecho Procesal Constitucional. diego. gamarra@ucu.edu.uy 
determina el reconocimiento de la posibilidad de distinguir, al menos en ocasiones, casos fáciles y difíciles. Se presenta, asimismo, una posición externa o interna sobre la discrecionalidad y una tesis fuerte y una débil sobre su configuración. Se sostendrá que desde una insoslayable mirada interpretativa -interna-, que supone además vocación de coherencia sostenible en la tarea, la discrecionalidad no lo es en un sentido fuerte sino excepcionalmente, pues supone argumentar sobre una solución excluyente de otras con fundamento en los materiales jurídicos que componen el sistema.

Palabras clave: interpretación jurídica, discrecionalidad de los jueces

ABSTRACT: On the grounds of Dworkin contributions, considering the inevitability of an interpretative perspective, a discreet conception of judicial discretion is proposed. It is herein accepted the thesis of the relative indeterminacy of language and, specifically, of rules -in the terms suggested by Hart- that, regarding adjudication of Law and with independence of the point of view, determines that the distinction among hard and soft cases is in general possible. Likewise, it is presented a strong and a weak thesis about discretion, taking also into account the internal and external perspective for such purposes. Finally, it will be defended that from an interpretative perspective, which is not possible to avoid and that supposes a task to be executed with vocation of coherency and stability, the discretion is only exceptionally strong, as interpretation of legal texts is carried out arguing about an excluding solution to be adopted and based on the recognized materials of the legal system.

KEY WORDS: interpretation of legal texts, judicial discretion

ReSUmo: No caminho desenhado por Dworkin, propõe uma concepção sobre a discrição jurisdicional-discreta-a partir do pressuposto de uma posição interpretativa necessária. A estes efeitos faz parte da tese da indeterminação parcial da língua e, em particular, das regras-de acordo com a formulação de Hart de tal posição, que em termos de aplicação da lei, independentemente da perspectiva, determina o reconhecimento da possibilidade de distinguir, pelo menos às vezes, casos fáceis e difíceis. Ele também apresenta uma posição externa ou interna sobre a discrição e uma tese forte e fraca sobre sua configuração. Argumentará que a partir de um olhar inevitável interpretativo-interno, que também implica uma vocação para a coerência sustentável na tarefa, a discrição não é em um sentido forte, mas excepcionalmente, porque implica discutir sobre uma solução exclusiva de outros baseados nos materiais jurídicos que compõem o sistema.

KEYWORDS: interpretación legal, discrição dos juízes 


\section{APERTURA DE LAS REGLAS E INDETERMINACIÓN PARCIAL}

Se asumirá a efectos de este trabajo la tesis de la indeterminación parcial del lenguaje, y específicamente de las reglas, reconociendo la existencia de zonas regladas y de discrecionalidad de los órganos encargados de aplicarlas, en última instancia de los órganos jurisdiccionales.

Tal postura se encuentra a medio camino entre las teorías escépticas de las reglas -de la indeterminación radical-y las negadoras de la indeterminación -que rechazan la discrecionalidad jurisdiccional-. Ante estas últimas opuestas y extremas tesis sobre la indeterminación, el «Caribdis» y el «Escila» de la teoría jurídica ${ }^{2}$, o bien la «pesadilla» y el «noble sueño» en la analogía onírica posteriormente propuesta por $\mathrm{Hart}^{3}$, la verdad se posicionaría en el medio. En continuación de la metáfora trazada por el profesor inglés, Moreso denomina a esta tesis de la indeterminación relativa como la «vigilia» ${ }^{4}$. Ni los jueces "crean» siempre exclusivamente y en forma irrestricta el Derecho, ni siempre en forma casi automática lo aplican sin que existan supuestos de «creación» jurisdiccional.

En grupos o comunidades numerosas, las pautas o normas generales ${ }^{5}$ son un instrumento básico de control social. Ellas comunican por referencia a clases de personas, de actos,

\footnotetext{
2 Herbert Hart, El Concepto de Derecho (Buenos Aires: Abeledo-Perrot LexisNexis Argentina S.A., Segunda Edición, 2007), 183.

3 Herbert Hart, "American Jurisprudence through English eyes: The Nightmare and the Noble Dream", en Essays in Jurisprudence and Philosophy (Oxford: Clarendon Press, 1983), 144. Véase también Nelson Ottonelli " Interpretación jurídica y decisión judicial: La indeterminación parcial del derecho y su carácter no neutral. Una búsqueda de vínculos entre las teorías de Hart y de Kennedy», en Revista Ruptura, Número 6, (Montevideo, Fundación de Cultura Universitaria, 2015), 203 y ss.

4 Juan José Moreso, La indeterminación del Derecho y la interpretación de la Constitución (Perú: Palestra. Segunda edición ampliada, 2014), 159 y 186 y ss.

5 Es común diferenciar entre generalidad y abstracción de las normas, sentando que en el primer caso se incluye una categoría indeterminada de sujetos y en el segundo una categoría indeterminada de casos. Un ejemplo de ello es la clasificación de actos administrativos incluida en el Decreto $\mathrm{N}^{\circ} 500 / 991$ del Poder Ejecutivo uruguayo. De aquí en más, en la medida en que la raíz de ambas categorías es la abstracción en un sentido amplio, se manejarán indistintamente.
} 
de cosas y circunstancias, a efectos de que sin necesidad de instruir caso a caso puedan ser comprendidas por muchos individuos como mandatos de comportamiento. Como indica Hart, sin esta posibilidad de formular mensajes generales y la capacidad de derivar de ellos mensajes particulares, nada de lo que se reconoce como Derecho podría existir ${ }^{6}$. En otras palabras, en la medida en que existen notorias limitaciones que impiden dirigir mensajes a todos los sujetos sobre todos los posibles casos particularmente, se apela a la capacidad humana de abstracción para que un ordenamiento jurídico en comunidades de cierta complejidad y extensión sea viable.

Así, en todos los campos de experiencia, en tanto acuden a un lenguaje, no sólo en lo que refiere a las reglas jurídicas, habrá casos particulares obvios en los que la derivación de una expresión general o su exclusión no resulta dudosa. Las palabras generales no servirían como instrumento de comunicación si no existieran casos familiares, no controversiales, un «núcleo de certeza», compuesto por las zonas de claridad y oscuridad, respectivamente de inclusión o exclusión indubitable. Pero habrá también otros casos en los que no resulte claro si dicha expresión les alcanza o no, la «zona de penumbra».

Como sostiene Hart, hay un límite a la orientación que el lenguaje abstracto puede efectivamente proporcionar y de presentarse ese punto en el que no existe convención firme sobre su sentido - por ejemplo, por repetirse constantemente en contextos semejantes- entonces el encargado de resolver tendrá que llevar a cabo una elección entre una serie de alternativas abiertas ${ }^{7}$. De ello se sigue un ámbito discrecional, que no deja de ser una elección, pero ello no significa, y es fundamental para la justificación del sistema que no signifique, que pueda ser irracional, infundada o arbitraria ${ }^{8}$.

El típico ejemplo que se reitera en los libros de introducción a la teoría del derecho para presentar en forma simple el problema es el de la prohibición de circular con vehículos en un parque. Nadie duda de que la prohibición comprende al tránsito en automóvil y que no comprende el tránsito peatonal, son casos claros, respectivamente de comprensión y exclusión de la regla, pero es dudoso si se encuentra o no vedado el uso de patines o de un triciclo.

No es diferente el análisis, si se toman casos en los que las normas generales contengan conceptos con una carga axiológica - de las que se suelen denominar principios-, incluso en casos constitucionales insignia, como las cláusulas que refieren con generalidad al derecho

\footnotetext{
Hart, El Concepto..., 155.

Hart, El Concepto..., 157 y 158.

Hart, El Concepto..., 159. Esta tesis de la racionalidad, parecería que no es otra cosa que la necesaria exigencia de una fundamentación de la opción escogida, que podría coincidir con versiones débiles de la llamada tesis de la respuesta correcta. Seguidamente se referirá con mayor dedicación a este punto.
} 
a la libertad de expresión o de prensa. Así, bajo la formulación referida, nadie dudaría que se encuentra permitido publicar información veraz que refiera a una red de narcotráfico manejada por el presidente de una república desde sus oficinas oficiales. Tampoco parece discutible que se encuentra vedado publicar videos alterados de individuos sin relevancia pública manteniendo relaciones sexuales en su hogar. Pero es bastante más dudoso si puede o no publicarse información veraz sobre el consumo de drogas por parte de un diputado en clubes nocturnos privados, fuera del ejercicio de sus funciones.

La interpretación de un determinado texto normativo, observado en su generalidad, es decir, no considerando el problema a partir de un caso sino de todos los casos que se comprenden bajo la regla, se encuentra acotada en la medida en que existen casos paradigmáticos, sin los cuales los términos en cuestión no tendrían eficacia comunicativa alguna.

Por su parte, si se realiza una interpretación en concreto, enfocada al cuestionamiento sobre un específico caso, aun cuando a priori pueda ubicarse en la zona de penumbra, una ejecución más detenida de los cánones de interpretación podría conducir en ocasiones a soluciones unívocas una vez que se enmarca el análisis.

De todas formas, es evidente que no puede afirmarse con certeza en todos los casos que de la interpretación se derive una solución incontrovertible. No sólo porque los sistemas no son siempre completos ${ }^{9}$, coherentes ${ }^{10}$ y precisos - por las dificultades que son propias del lenguaje y, además, porque son diseñados por hombres y a lo largo del tiempo-, sino, entre otras cosas, porque suele también discreparse sobre los métodos de interpretación y de aplicación ${ }^{11}$, pese a que con relativo consenso se los identifique como instrumentos atendibles, en buena medida dirigidos a configurar el sistema en términos de racionalidad.

\footnotetext{
9 Podrían serlo si se introduce una cláusula de clausura, como el principio de libertad de los individuos. Véase Luis Prieto Sanchís, Justicia constitucional y derechos fundamentales (Madrid: Edit. Trotta, 2009), 249 y ss. En verdad sería también discutible en esos casos la propia completitud, pero no cabe aquí explayarse en el punto.

${ }_{10}$ No sólo en el sentido lógico normativo -referente a las antinomias- como el que proponen Alchourrón y Bulygin, sino, también como unidad de sentido y de fin que se derivaría de un ordenamiento con pretensión de establecer soluciones igualitarias a los casos. Nótese que la propia igualdad o coherencia -que podría consagrarse normativamentetambién es, en tanto referente genérico, objeto de zonas de certeza y de dudas.

11 Una buena muestra de lo indicado es el relevamiento que realiza Sagüés de la alternancia en el empleo de criterios interpretativos por la Corte Suprema de Justicia argentina. No sólo se discrepa sobre los criterios interpretativos sino que indiscriminadamente se alterna en su utilización. Néstor Pedro Sagüés, «Interpretación constitucional y alquimia constitucional (El arsenal argumentativo de los tribunales supremos)", en Revista Iberoamericana de Derecho Procesal Constitucional, No 1, (2004). Véase una buena crítica a la situación referida en Roberto Gargarella, «De la alquimia interpretativa al maltrato constitucional. La interpretación de la Constitución en manos de la Corte Suprema Argentina», en Biblioteca Jurídica Virtual del Instituto de Investigaciones Jurídicas de la UNAM, https://archivos.juridicas.unam.mx/ www/bjv/libros/7/3015/6.pdf, 53 y ss.
} 


\section{APERTURA, INTERPRETACIÓN Y DISCRECIONALIDAD INTERNA EN SENTIDO DÉBIL Y FUERTE}

De acuerdo con lo indicado previamente, pueden existir casos difíciles, es decir, casos en los que se presentan verdaderas dudas para identificar si una regla general los comprende o, en otras palabras, para determinar si una regla particular que provea su solución en un sentido pertenece al sistema por derivación de una norma general. En dichos casos, ante su planteamiento jurisdiccional, se afirmó sin mayores explicaciones que el juez posee discrecionalidad. Ahora bien, es necesario profundizar la pesquisa, cuestionarse sobre los casos difíciles y sobre el alcance de la discrecionalidad y para ello se analizará el asunto desde una doble perspectiva, una externa y una interna.

Por cierto, el límite entre los casos difíciles y los casos fáciles constituye en sí mismo un caso difícil. Presentado el problema de una manera simpática -al menos pretendidamente-, cabe indicar que ello se debe a que la vaguedad es vaga -técnicamente, a la vaguedad de segundo orden $-{ }^{12}$. ¿En qué momento puede con certeza afirmarse que una solución no es en lo absoluto controvertible? La interrogante parece poner en aprietos la tesis de la indeterminación parcial, pero en tanto también sabemos que la vaguedad algo es pese a ser vaga y que nos comunicamos razonablemente apelando a su uso y a términos que la revisten, verdaderamente no se la compromete ${ }^{13}$. Aun así, resta analizar cómo se llega a determinar que un caso es difícil y para ello debe considerarse el paso de la interpretación de los textos normativos, pues la tarea de atribución de significado es precisamente la que resulta sensible a las dudas sobre el alcance de una regla.

Si un observador externo del funcionamiento del sistema se cuestiona sobre la dificultad de un caso, no podría obtener una respuesta sino apelando a recabar información de los operadores - de los participantes de la práctica-, por ejemplo, analizando cómo se resuelven casos similares por diferentes magistrados, realizando experimentos con casos similares y registrando reacciones, encuestando abogados, partes litigantes, académicos y jueces. Ello puede tener una utilidad, pero también interesa el problema desde la perspectiva de estos últimos, la interna, y la protagonista desde esas tiendas es la interpretación.

12 María Cristina Redondo, "Teorías del Derecho e indeterminación normativa», DOXA, N. 20, (1997), 191.

13 Véase Moreso, La indeterminación..., 160 y 161 y Claudina Orunesu, Positivismo jurídico y sistemas constitucionales (Madrid, Barcelona, Buenos Aires: Marcial Pons, 2012), 30 y ss. 
Puede externamente concluirse que cierto caso es difícil y la discrecionalidad asociada a la indeterminación presentarse como la posibilidad que un juez tiene de resolverlo en un sentido o en otro sin ser responsabilizado. Sin embargo, la constatación de la práctica no será del todo completa en tanto no se tome en cuenta el fenómeno interpretativo como paso previo en una práctica continua y sostenida.

Podría replantearse el punto indicando que el juez, ante las dudas sobre el Derecho y la existencia de criterios interpretativos alternativos que sean razonablemente admitidos, puede resolver en el sentido que prefiera siempre que argumente a partir de ciertas bases formales y en función de algunos de esos criterios. Presentando así la solución arribada como la mejor a partir de los materiales jurídicos de los que parte, o bien simplemente como una posible solución en caso de desempate ante alternativas de idéntica bondad conforme una interpretación consistente.

Asumiendo entonces esta visión ajustada de la mirada externa, considerando que el Derecho requiere inevitablemente de interpretación, es más preciso explicar el problema no como un caso en el que el juez "puede» sin ser responsabilizado, sancionado o fuertemente criticado por la generalidad de practicantes resolver en cualquier sentido; sino como uno en el que efectivamente opta entre criterios razonables y efectúa una argumentación que plausiblemente conduce a una solución, pese a que otras argumentaciones también razonables, aunque a su juicio menos convincentes, podrían conducir a otras soluciones.

En el dominio interno, parecería que cuando se efectúa una interpretación y no otra, en principio no se lo hace caprichosamente ni apelando al subjetivismo más descarnado. Las decisiones parecen tomarse y respaldarse en virtud de una determinada teoría o postura sobre el sistema de fuentes - de adhesión inevitablemente subjetiva pero no descarnada- que en mayor o menor medida supone su apego y cuya explicitación es debida en términos jurídicos. En un terreno descriptivo, que debería corroborarse empíricamente, intuyo que la solución efectivamente tiende a ser la indicada. En un terreno prescriptivo, entiendo que así debería ser.

Debería todavía indagarse más y distinguirse si quien juzga parte de una solución y construye una argumentación razonable, descartándola eventualmente en los casos en los que no resulte posible sustentarla, o si se efectúa una interpretación desde determinadas posturas con vocación de coherencia que conducen a la solución ${ }^{14}$. Más aún, cabría a su vez

\footnotetext{
14 Tan complejo es definirlo que Zagrebelsky, que además de ser un destacado jurista fue magistrado de la Corte Constitucional italiana, indica que se parte del caso -no me queda claro si se refiere a una idea sobre su solución- y que luego se busca en las reglas para volver a él en un procedimiento bipolar que adecúa las exigencias del caso - ¿una idea sobre su resolución?- y las pretensiones de las reglas -¿qué pretensiones?-. Véase Gustavo Zagrebelsky, El derecho dúctil. 
cuestionarse si en el primer caso indicado, que se enfoca de plano en la solución, ello es gestado por una "preconcepción" -que es en última instancia interpretativa, aunque pasada-, si es meramente una reacción emotiva, una opción por una moral subjetiva pura y dura o si, como pareciese ser, responde, según el caso, a una combinación de tales causas con diferente peso.

Un caso difícil en un sentido muy estricto - de dudas insuperables-, debería considerarse internamente al final del camino, toda vez que un operador, luego de interpretar un texto normativo, considerando las disposiciones pertinentes y su contexto con afán de coherencia, concluya genuinamente -todo lo que su naturaleza humana le permita- que existen argumentos de idéntico peso para concluir que no existe ni puede derivarse del sistema una regla que establezca una solución u otra para un caso. La discrecionalidad en estos casos lo es en un sentido fuerte, en la medida en que el aplicador del Derecho sentirá que puede resolver, sin más, en cualquier sentido.

Pero el trecho interpretativo que permite llegar a esa extraña situación, para quienes trabajamos con el Derecho resulta realmente largo. Si se parte del comienzo del camino y no de su final, lo cierto es que se abordará el sistema normativo para intentar despejar las dudas -que por lo general las habrá y muchas- y solucionar el caso de la forma que resulte más consistente en función de los materiales jurídicos disponibles. Desde esta otra vereda, la discrecionalidad se ve de manera diferente, como un escenario de alternativas preliminares ligado al cumplimiento de un «deber» de justificar la opción por la solución más convincente en función de una argumentación jurídica que presente al sistema de la forma más coherente -discrecionalidad en sentido débil- ${ }^{15}$. Ello no implica desconocer el infranqueable límite de la excepcional paridad al final del camino, insisto, en cuyo caso la discrecionalidad lo es forzosamente en sentido fuerte, pese a que por razones pragmáticas y morales pueda incluso discutirse si conviene o no explicitarlo.

En la mayoría de los casos no puede con sinceridad afirmarse que una respuesta no admita dudas o que sea inequívoca, lo que equivale a afirmar que son al menos dos las soluciones posibles conforme interpretaciones razonables de materiales del ordenamiento. Empero, en la medida en que por lo general es posible que se perciban posturas más razonables que otras, fundamentalmente al cotejar argumentaciones y las bases escogidas para formularlas a partir de una idea regulativa de coherencia y decisiones pasadas -pues denota

\footnotetext{
Ley, derechos, justicia (Madrid: Editorial Trotta, Séptima Edición, 2007), 133 y 134. Del mismo autor: La ley y su justicia. Tres capitulos de justicia constitucional, (Madrid, Editorial Trotta, 2014), 201 y ss.

${ }_{15}$ Sobre el sentido débil y fuerte de la discrecionalidad véase Ronald Dworkin, Los derechos en serio, (Barcelona: Planeta-Agostini, Barcelona, 1993), 84, 85, 452 y 453. Título original en inglés, Taking Rights Seriously, 1977.
} 
arbitrariedad un cambio de posición sin explicaciones suficientes-, en casos a priori borrosos no es atinado afirmar que el asunto pueda resolverse de cualquier manera, es decir, en forma azarosa o, como con ingenio plantea Rodríguez Carrau, tirando una moneda ${ }^{16}$.

El punto de inflexión para admitir prescriptivamente la discrecionalidad en sentido fuerte desde una perspectiva interna no puede ser cualquier vacilación en el proceso interpretativo. Entre otras cosas, porque no hay razones para resignar la búsqueda de una solución que al magistrado le resulta impuesta con base en determinadas fuentes que conforman un sistema con pretensión de consistencia, siendo que lo que en parte está en juego es la propia calificación del caso como fácil o difícil ante una barrera de ubicación dinámica.

\section{RECAPITULACIÓN DEL ANÁLISIS EN TÉRMINOS DE CUESTIONAMIENTOS SOBRE LA ÚNICA RESPUESTA CORRECTA}

Resta relacionar estas reflexiones con las tesis de la única respuesta correcta, pues no puede esconderse su conexión, para luego sí, finalmente, ordenar un poco las ideas en forma sintética. Las posiciones sobre la única respuesta correcta, esto es, que analizan si necesariamente existe siempre una única solución jurídica para un caso, pueden ordenarse gradualmente. En un extremo es posible ubicar a quienes la consideran indiscutible en tanto se puede extraer de los principios del derecho natural y en el otro a quienes la niegan radicalmente postulando que tal idea no es más que un recurso ideológico para enmascarar el poder político de los jueces.

Es posible identificar entre las posiciones indicadas unas cuantas alternativas interme$\operatorname{dias}^{17}$, entre las que destaca la propuesta originalmente por Dworkin. El autor norteamericano sostiene que la respuesta correcta existe en tanto el Derecho -como práctica- además de reglas se compone de valores y principios, y la solución adecuada para un caso consiste en la mejor interpretación posible de los materiales jurídicos en función de los valores y principios referidos, que contribuyen a presentar a la práctica jurídica «bajo su mejor luz». Si se prefiere, conforme una teoría moral y política adecuada, que tenga en cuenta el desarrollo de la prác-

\footnotetext{
16 Guzmán Rodríguez Carrau, «No tirarás la moneda: términos jurídicos, vaguedad y el deber de los jueces de juzgar, pase lo que pase», en Revista Ruptura, Número 3, (Montevideo: Fundación de Cultura Universitaria, 2012).

Manuel Atienza, «Sobre la única respuesta correcta», en Aulis Aarnio, Manuel Atienza y Francisco J. Laporta, Bases teóricas de la interpretación jurídica, (Madrid: Fundación Coloquio Jurídico Europeo, 2010), 56 a 59.
} 
tica y que dote de consistencia a los materiales ${ }^{18}$. No se pretende aquí recoger la tesis dworkiniana de la respuesta correcta en su totalidad, que se conecta con otra serie de postulados que no se comparten, como la existencia de principios como estándares diferentes de las normas en sentido estricto, ni de admitir las posiciones epistemológicas que asume. Sin embargo, se suscribe sí el sustrato de su gran aporte, la importancia de no omitir la perspectiva interna y concebir el Derecho interpretativamente y como integridad, orientado a fines.

En forma algo menos ambiciosa y sin contienda con la tesis de la indeterminación parcial conviene destacar lo siguiente:

(i) Que pese a que existen casos claros y difíciles el límite es impreciso ${ }^{19}$, potencialmente cambiante y que para ubicarlo se requiere de una interpretación. De ello se sigue que el decisor debe en cualquier caso justificar su decisión en los materiales del sistema con afán de consistencia lógica y teleológica -puede que haya más casos claros de los que parecen y las confusiones resulten superfluas-.

(ii) Que desde una perspectiva interna efectivamente se parte de una convicción teórica sobre el sistema y su interpretación -técnica-, y que las decisiones adoptadas sobre esas bases sientan un punto de referencia para futuras decisiones -el cambio es posible, pero exige fundamentación-. Desde esos postulados es que se determinan soluciones a casos concretos descartando por lo general otras menos convincentes. La discrecionalidad lo será predominantemente en un sentido débil.

(iii) Que en la medida en que es posible que un intérprete efectivamente concluya que un caso puede no tener solución, entonces sí existirá discrecionalidad en sentido fuerte, aunque parece algo excepcional ${ }^{20}$.

(iv) Que, partiendo de la discrecionalidad desde una perspectiva externa, no parece adecuado extraer conclusiones más allá de lo que se observa y que debe observarse el fenómeno en forma completa para que sean formuladas debidamente. Si bien es cierto que

18 Ronald Dworkin, «¿Puede ser que no haya una respuesta correcta para los casos difíciles?», en Una cuestión de principios, (Buenos Aires: Siglo Veintiuno Editores, Colección derecho y política, 2012), págs. 159 y ss. Véase del mismo autor también El imperio de la justicia. De la teoría general del derecho, de las decisiones e interpretaciones de los jueces y de la integridad política y legal como clave de teoría práctica, (Barcelona: Edit. Gedisa, 2008), 164 y 165.

19 En similar sentido, evidenciando dificultades de la distinción, Streck indica que no se tiene en cuenta la existencia de un acontecer en el precomprender, en el cual el caso simple y el caso difícil se enraízan. Lenio Luiz Streck, Verdad y consenso. De la posibilidad a la necesidad de respuestas correctas en Derecho, (Montevideo, Buenos Aires: Editorial BdeF, 2012), 284 y 285.

20 Véase Tomás Ramón Fernández y Alejandro Nieto, El Derecho y el revés (Barcelona: Editorial Ariel, 2000), 136, Tomás Ramón Fernández, "Respuesta a Manuel Atienza», en Revista DOXA, número 23 (2000), 756, 759 y 760. Allí el prestigioso administrativista sostiene que en treinta ańos de ejercicio únicamente en un caso consideró que dos soluciones eran igualmente correctas, es decir, que el problema no tenía solución. Manuel Atienza, "Sobre la única respuesta correcta», 71 y 72. Del mismo autor: «El sentido del Derecho. Carta a Tomás Ramón Fernández», en $D O X A$, N. 23, (2000), 737 y ss. 
dos magistrados pueden resolver un idéntico caso de forma distinta sin incumplir ninguna norma, no lo es que no incumplan ninguna norma si no argumentan hasta las últimas consecuencias y bajo una teoría coherente las razones de su decisión a partir de una interpretación consistente de los materiales jurídicos. En los sistemas modernos las decisiones infundadas son ilegítimas en tanto pueden determinar su invalidez y desencadenar responsabilidad y sanciones para el decisor.

(v) La posibilidad de utilizar la «maleabilidad» $\mathrm{o}$ «ductilidad» del Derecho ${ }^{21}$-en puridad en las zonas de penumbra- para esconder bajo argumentaciones lo que caprichosamente el intérprete por alguna razón prefiere es en cierto punto inevitable, pero no es necesariamente la forma en la que los jueces generalmente actúan. Aunque se llegue a reconocer que primero avizoran la solución del caso, ello puede en buena medida deberse a una preconcepción de un sistema que les es familiar - previamente interpretado conforme unos presupuestos teóricos y técnicos consistentes- y no a su arbitrio a secas luego camuflado ${ }^{22}$. En cualquier caso, si hay algo que es claro en todo esto es que dichas notas psicológicas y su peso más o menos determinante para la resolución de un caso no son de fácil medición.

Si quisiera catalogarse el planteo formulado bajo una postura en relación con la respuesta correcta, que insisto es confuso por sus múltiples aristas, pero absolutamente frecuente, debería concluirse que ésta no es más que un ideal regulativo desde la perspectiva interna ${ }^{23}$, apoyado en un afán de coherencia y conducido por un método que, al menos en ese aspecto básico de racionalidad, parece generalmente admitido y acota -en ocasiones más y en ocasiones menos- el margen de apreciación.

Considerada como un resultado efectivo, en el marco de la admisión de una indeterminación parcial del Derecho y de la posibilidad de análisis desde una perspectiva interna y externa, la respuesta correcta es de hallazgo contingente. Aparentemente preponderante desde la mirada del intérprete por las razones antedichas, y más excepcional desde una mirada externa incompleta, esto es, conforme una verificación de casos unívocamente resueltos por la generalidad de los operadores.

${ }^{21}$ Según la terminología de Gustavo Zagrebelsky. Véase Gustavo Zagrebelsky, El derecho dúctil..., 14. Del mismo autor, La ley y su justicia..., 226.

22 En el mismo sentido Herbert Hart, El Concepto de Derecho..., 175.

23 Véase en similar sentido Robert Alexy, "Sistema Jurídico, Principios Jurídicos y Razón Práctica», en $D O X A$, N. 5 (1988), 151. "La idea regulativa de la única respuesta correcta no presupone que exista para cada caso una única respuesta correcta. Sólo presupone que en algunos casos se puede dar una única respuesta correcta y que no se sabe en qué casos es así, de manera que vale la pena procurar encontrar en cada caso la única respuesta correcta. Las respuestas que se encuentren, en el marco de este intento, sobre la base del nivel de la regla y de los principios, de acuerdo con los criterios de la argumentación jurídica racional, que incluyen los de la argumentación práctica general, también responden entonces, aunque no sean las únicas respuestas correctas, a las exigencias de la razón práctica y, en este sentido, son al menos relativamente correctas». 
Para citación: Gamarra Ante, Diego, La mirada interpretativa como base de una discrecionalidad discreta, en Revista de Derecho No 19 (jul.-2019), ISSN 1510-3714, ISSN On line 2393-6193, 181-193

Contribución 100\% Diego Gamarra Ante.

\section{BIBLIOGRAFÍA}

Alexy, Robert, «Sistema Jurídico, Principios Jurídicos y Razón Práctica», DOXA, N. 5 (1988). Atienza, Manuel, «Sobre la única respuesta correcta», en Aulis Aarnio, Manuel Atienza, Francisco J. Laporta, Bases teóricas de la interpretación jurídica, Madrid: Fundación Coloquio Jurídico Europeo, 2010.

Atienza, Manuel, «El sentido del Derecho. Carta a Tomás Ramón Fernández», DOXA, N. $23(2000)$.

Dworkin, Ronald, Los derechos en serio, Barcelona: Planeta-Agostini, 1993.

Dworkin, Ronald, «¿Puede ser que no haya una respuesta correcta para los casos difíciles?», en Una cuestión de principios, Buenos Aires: Siglo Veintiuno Editores, 2012.

Dworkin, Ronald, El imperio de la justicia. De la teoría general del derecho, de las decisiones e interpretaciones de los jueces y de la integridad politica y legal como clave de teoría práctica, Barcelona: Edit. Gedisa, 2008.

Fernández, Tomás Ramón, «Respuesta a Manuel Atienza», DOXA, N. 23 (2000).

Fernández, Tomás Ramón y Nieto, Alejandro, El Derecho y el revés, Barcelona: Editorial Ariel, 2000.

Gargarella, Roberto, «De la alquimia interpretativa al maltrato constitucional. La interpretación de la Constitución en manos de la Corte Suprema Argentina», UNAM, https:// archivos.juridicas.unam.mx/www/bjv/libros/7/3015/6.pdf.

Hart, Herbert, El Concepto de Derecho, Buenos Aires: Edit. Abeledo-Perrot LexisNexis Argentina S.A, 2007.

Hart, Herbert, «American Jurisprudence through English eyes: The Nightmare and the Noble Dream», en Essays in Jurisprudence and Philosophy, Oxford: Clarendon Press, 1983.

Moreso, Juan José, La indeterminación del Derecho y la interpretación de la Constitución, Perú: Palestra, 2014. 
Orunesu, Claudina, Positivismo jurídico y sistemas constitucionales, Madrid, Barcelona, Buenos Aires: Marcial Pons, 2012.

Ottonelli, Nelson, «Interpretación jurídica y decisión judicial: La indeterminación parcial del derecho y su carácter no neutral. Una búsqueda de vínculos entre las teorías de Hart y de Kennedy", Revista Ruptura (FCU), N. 6 (2015).

Prieto Sanchís, Luis, Justicia constitucional y derechos fundamentales, Madrid: Edit. Trotta, 2009.

Redondo, María Cristina, «Teorías del Derecho e indeterminación normativa», DOXA, N. 20 (1997).

Rodríguez Carrau, Guzmán, «No tirarás la moneda: términos jurídicos, vaguedad y el deber de los jueces de juzgar, pase lo que pase», Revista Ruptura, N. 3 (2012), FCU.

Sagüés, Néstor Pedro, «Interpretación constitucional y alquimia constitucional (El arsenal argumentativo de los tribunales supremos)», Revista Iberoamericana de Derecho Procesal Constitucional, No 1 (2004).

Streck, Lenio Luiz, Verdad y consenso. De la posibilidad a la necesidad de respuestas correctas en Derecho, Montevideo, Buenos Aires: Editorial BdeF, 2012.

Zagrebelsky, Gustavo, El derecho dúctil. Ley, derechos, justicia, Madrid: Editorial Trotta, Séptima Edición, 2007.

Zagrebelsky, Gustavo, La ley y su justicia. Tres capitulos de justicia constitucional, Madrid: Editorial Trotta, 2014. 